\title{
Educación superior y competencias para el empleo. El punto de vista de los empresarios ${ }^{1}$
}

\section{Higher education and employment related skills. The point of view of employers}

\author{
M. Teresa BARTUAL FIGUERAS y Joaquín TURMO GARUZ \\ Universidad de Barcelona
}

Recibido: Enero 2015

Evaluado: Marzo 2015

Aceptado: Abril 2015

\begin{abstract}
Resumen
La relevancia del conocimiento como input del proceso productivo ha aumentado la complejidad de la contratación en el mercado de trabajo cualificado. Como consecuencia de ello, se ha generado un proceso de reflexión sobre la adecuación de la acreditación universitaria a las necesidades del mercado de trabajo. Académicos, gerentes y expertos en el mercado de trabajadores altamente cualificados han tenido parte en esta reflexión durante mucho tiempo. Este trabajo tiene como objetivo la identificación de las competencias profesionales con mayor relevancia en la empleabilidad de los graduados en Economía y Empresa. El análisis se basa en una investigación cualitativa que toma como fuentes de información las opiniones de los empleadores. La información para el estudio ha sido obtenida mediante entrevistas en profundidad y la realización de un grupo de discusión. En este proceso han participado empresarios, responsables del servicio de prácticas de la Universidad de Barcelona, expertos y responsables de empresa de colocación, representantes de organizaciones empresariales y profesores universitarios. La atención se centra en la percepción que los entrevistados tienen del requerimiento de conocimientos, habilidades y actitudes, en el grado en que los desarrollan y en los cambios que se necesitarían para lograr una mejor correspondencia entre las competencias adquiridas por los graduados y las requeridas por el mercado de trabajo. A partir de la clasificación de las competencias profesionales (proyecto Tuning), el estudio pone de relieve la importancia otorgada por los empleadores a de las competencias genéricas. No obstante, se observan diferencias valorativas según la tipología de empresas. Asimismo, se evidencian déficits en algunos aspectos relevantes, como la formación práctica y la capacidad de iniciativa, de análisis o de organización. Por último, de las opiniones recogidas también se constata la necesidad de aproximar la universidad al sistema productivo, al menos en el campo económico-empresarial.
\end{abstract}

Palabras clave: educación superior, competencia profesional, empleo, graduados en administración y dirección de empresas.

\footnotetext{
${ }^{1}$ Este trabajo ha contado con la ayuda del Instituto de Ciencias de la Educación de la Universidad de Barcelona a través del Programa de Investigación en Docencia Universitaria (REDICE-14-1324) 


\begin{abstract}
The relevance of knowledge as an input in the productive process has increased the complexity of the process of hiring in the market of qualified work. As a consequence, a process of reflection on the adequacy of university accreditation to the needs of the labor market has generated. Academics, managers and experts in labor market of highly skilled workers have taken part in this reflection for a long time. This paper aims to identify the most relevant professional competencies in the employability of graduates in Economics and Business. The analysis is based on a qualitative research which adopts the opinions of employers as its source of information. This information has been obtained through thorough interviews and a discussion group. Entrepreneurs, managers of student practices of the University of Barcelona, labor market, experts, representatives of business organizations, and academics, have participated in the process. Attention is focused on the perception of the interviewees regarding the required knowledge, skills and attitudes, the extent to which developed those abilities and the changes that would be needed to achieve a better match between the acquired competencies and those required by the labor market. Taking into account the classification of skills carried out in this study (Tuning Project), we conclude that employers particularly value generic skills. However, there are evaluative differences depending on the type of companies. In addition, deficiencies are also evidenced in relevant aspects, such as practical training and in initiative, analytical or organization skills. Lastly, opinions of respondents also suggest that it would be convenient to bring closer the Universities and enterprises, at least in the economic and business fields.
\end{abstract}

Keywords: higher education, professional skills, employment, business administration graduates.

Durante las últimas décadas, la evolución del mercado de trabajo ha estado marcada por la dinámica del cambio técnico en la denominada sociedad del conocimiento. Esto ha conllevado cambios en la organización y métodos de trabajo, así como en la demanda de necesidades formativas. La vinculación entre la adecuación de la acreditación universitaria y la carrera laboral de los graduados se ha vuelto más compleja, demandándose perfiles más amplios y flexibles. La respuesta a estas exigencias supuso la apertura de un proceso de reflexión sobre la idoneidad de la formación universitaria y la empleabilidad de los graduados, que desembocó en la firma de la Declaración de Bolonia en 1999. Dicha declaración fue el punto de partida de un proceso en el que se sentaron las bases de una reforma estructural de la Educación Superior a nivel europeo (el Espacio Europeo de Educación Superior). El objetivo de este proceso era aproximar la formación académica, en conocimiento y habilidades, a los requerimientos que la sociedad del conocimiento demandaba. Se incidía, también, en una nueva orientación del modelo de enseñanza-aprendizaje, acorde con un enfoque integrado y global, orientado al desarrollo de competencias, tomando como referente principal al estudiante (González y y Wagenaar, 2003).

Empleadores y graduados se han visto implicados en estos cambios experimentados en la universidad y en el mercado de trabajo cualificado. A partir de diversas observaciones sobre ambos colectivos, una larga serie de trabajos han intentado contrastar el ajuste entre los requisitos de la oferta y las condiciones de demanda de este mercado. En el ámbito europeo podemos destacar: Careers After Higher 
Education: A European Reserarch Study (CHEERS) The flexible Professional in the Knowledge Society (REFLEX), publicados en el años 2000 y 2005, respectivamente, o los trabajos de Schomburg y Teichler (2006), Teichler (2007), Andrew y Hgison (2008) García-Aracil y Van der Velden (2008), Tomlinson (2012) o Abdullah et al (2014), entre otros. En el ámbito español, destacan los estudios de la Agencia Nacional de la Evaluación y Acreditación de la educación Superior (ANECA) o los de las Agencias Regionales, así como los trabajos de Alonso et al (2008), Marzo-Navarro et al. (2009), Corominas et al. (2010), Freire et al. (2013), Montoro-Sánchez et al. (2012), Martín del Peso et al. (2013), o Rodriguez (2015), entre otros. Desde diferentes enfoques, estos estudios coinciden en la relevancia de las competencias genéricas, ligadas a la personalidad y desarrollo cognitivo, así como en la existencia de desajustes entre el nivel competencial requerido por las empresas y el que presentan los titulados aspirantes a ocupar un puesto de trabajo. En consecuencia, consideran necesario aproximar la formación superior a las necesidades de la sociedad del conocimiento. Partiendo de una reflexión sobre el concepto de competencias profesionales en el marco de la sociedad del conocimiento, este trabajo pretende identificar las competencias profesionales más relevantes en la empleabilidad de los graduados en economía y empresa. El análisis se basa en una investigación cualitativa que toma como fuente de información las opiniones de empleadores y expertos en colocación, centrando la atención en la percepción que los entrevistados tienen del requerimiento de conocimientos, habilidades y actitudes, el grado en el que los desarrollan y los cambios que se necesitarían para lograr una mejor correspondencia entre oferta y demanda.

El trabajo se estructura en cinco epígrafes. El segundo epígrafe introduce una reflexión sobre conocimiento, competencias y empleabilidad. El tercero expone la metodología cualitativa empleada. En el cuarto se analizan los resultados del análisis. Finalmente, en el último epígrafe se detallan las conclusiones.

\section{Conocimiento, competencias y empleabilidad.}

El conocimiento como input incorporado al trabajo ha sido creciente y acumulativo a lo largo de la historia (Trullén et al., 2002); de manera que en la segunda mitad del siglo XX el análisis económico lo incorporó como input en el proceso productivo, por lo tanto, como factor relevante en el aumento de la productividad (Schultz, 1961, Romer 1990).

Esta relevancia del conocimiento en la actividad económica y su repercusión en el mercado de trabajo determinaron el interés por el análisis de las condiciones de demanda y oferta de trabajo cualificado. Así, las teorías sobre el mercado de trabajo inciden en diversos aspectos que pretenden explicar su funcionamiento: la relación entre la inversión en formación y las expectativas de remuneración de los profesionales, que establece la teoría del capital humano (Mincer, 1958; Becker, 1964); la asimetría de información existente entre demandantes y oferentes de trabajo (Spence, 1973); y otros aspectos como el nivel de formación de los trabajadores como 
señal de referencia para la demanda de trabajo por parte de las empresas (Thurow, 1983).

De los tres aspectos mencionados, la asimetría en la información entre demandantes y oferentes de trabajo cualificado constituye el centro de atención del presente trabajo, dado que es uno de los elementos que más dificulta contratación de los graduados universitarios. En efecto, la exigencia de adaptación a los cambios, junto a la creciente diversidad de conocimientos y habilidades que precisa el proceso productivo, han aumentado la complejidad en la identificación de las competencias de los oferentes de trabajo; esto ha provocado un aumento de la incertidumbre en el proceso de selección y contratación. En consecuencia, se ha hecho más difícil el ajuste entre oferta y demanda de graduados, hasta el punto de que la propia titulación ha ido perdiendo relevancia como señal de referencia que acredita conocimientos de los graduados en el mercado (Spence, 1973; Tomlinson, 2012).

La preocupación por la definición de las competencias profesionales viene determinada por la necesidad de analizar y catalogar la aplicación del conocimiento a la actividad productiva. Un punto de partida para dicho análisis ha sido la distinción entre el conocimiento codificado, transmitido mediante la formación institucionalizada, y el conocimiento tácito, más relacionado con las características intrínsecas de la persona (Polany, 1958). Esta distinción metodológica hace necesario introducir nuevos atributos en el análisis del mercado laboral de los graduados, además de los que se refieren simplemente al conocimiento específico o técnico necesario para realizar las tareas propias del puesto de trabajo. Se trata de los atributos transversales, relacionados con aspectos de la personalidad o de la experiencia, como el talento, la responsabilidad, la capacidad de relación y comunicación, etc. (Heckman et al., 2006). Todos ellos conforman las competencias profesionales, mediante las que se pretende abarcar el amplio espectro de requerimientos necesarios para el desarrollo del trabajo cualificado.

En definitiva, el estudio de las competencias profesionales es el análisis de las características del conocimiento y las habilidades de los trabajadores, desde el punto de vista de la orientación práctica de la actividad profesional. Para los empleadores, las competencias constituyen un elemento metodológico en la determinación de los requerimientos que precisan en los graduados.

En este sentido, el término competencia engloba el conjunto de conocimientos, habilidades y actitudes que describen la capacidad de un graduado para responder a las exigencias de su puesto de trabajo (Biemans, et al, 2004; González y Wageneaar, 2003). Su clasificación permite diferenciar entre competencias específicas y competencias genéricas. Las primeras hacen referencia a los conocimientos y capacidades técnicas requeridas para desarrollar una actividad profesional; engloban aquellas competencias relacionadas con las disciplinas académicas y se adquieren, mayoritariamente, mediante la formación o la experiencia. Las competencias genéricas son competencias transversales que aluden, como se ha mencionado, a las habilidades relacionadas con la personalidad, la actitud y la cultura, incluyen aspectos como el pensamiento crítico y ético, la capacidad de análisis y síntesis, la de planificación 
estratégica, adaptación, el liderazgo, la motivación, la responsabilidad o la capacidad de relación y comunicación.

\section{Aspectos metodológicos}

Para la obtención de la información a analizar se ha considerado conveniente el uso de una metodología cualitativa, basada en la realización de entrevistas semiestructuradas, que se complementaron mediante un grupo de discusión con tres ámbitos de interacción diferenciados; todo ello sobre el mismo objeto de estudio (Vela, 2001; Brown, 2010; Corbetta, 2010). Esta metodología, pese a las limitaciones de representatividad que puede entrañar en determinados casos (Pérez, 2005), permite obtener la información y el rigor científico suficientes, especialmente si entrevistador y entrevistado comparten un grado de conocimiento similar sobre el tema de estudio, como ocurre en este caso. Las entrevistas se confeccionaron en torno a cuatro grandes temas, predeterminados a partir de varias sesiones de trabajo:

- El análisis, por parte de los empresarios, de las competencias de los graduados.

- La percepción de dichas competencias según la dimensión de la empresa.

- Los desajustes cualitativos entre oferta y demanda de competencias.

- El papel de la Universidad y la relación con la empresa.

La selección de los entrevistados y el grupo de discusión se realizó siguiendo el criterio del muestreo teórico (Patton, 2015). Se alcanzó el nivel de saturación una vez realizadas doce entrevistas, de las catorce totales. Tanto en las entrevistas como en el grupo de discusión hubo suficiente representación de los diversos tipos de empresas, representantes del ámbito académico y de la Administración Pública, como puede observarse en el anexo.

Las entrevistas se realizaron entre enero y junio de 2013 y tuvieron una duración aproximada de noventa minutos; siendo todas ellas grabadas y transcritas.

El grupo de discusión estuvo formado por una mesa de trabajo liderada por cuatro personas (un representante de la Universidad de Barcelona, el director de Operaciones de una empresa de colocación, un empresario con experiencia en pequeñas y medianas empresas y una consultora del Área de Derecho del trabajo de una empresa multinacional) y un foro de más de cincuenta asistentes, donde se identificaron tres grupos de discusión: representantes de los estamentos académicos, de la empresa y de la Administración Pública (ver anexo). La discusión se efectuó en noviembre de 2013, siguió un guion abierto y tuvo una duración de cuatro horas.

Las opiniones vertidas en las entrevistas y en el grupo de discusión han sido analizadas y calibradas por los autores para adecuarlas a la tipología de las competencias profesionales basada en el proyecto Tuning-Europa (González y Wagenaar, 2003 $)^{2}$. La información se analizó de acuerdo con los cuatro grandes temas

\footnotetext{
${ }^{2}$ El proyecto clasifica las competencias en genéricas y específicas; dentro de las genéricas distingue entre instrumentales (habilidades ligadas a capacidades cognitivas, metodológicas y lingüísticas), 
mencionados, de modo que permitiera la clasificación de las opiniones, así como su codificación y sistematización.

\section{Resultados del estudio. La visión de los empleadores}

En este epígrafe se exponen y analizan los resultados de las entrevistas agrupados de acuerdo con el tipo de competencia a que hacen referencia, según la clasificación Tuning anteriormente mencionada. También se exponen y analizan las opiniones que los agentes entrevistados expresaron sobre el papel de la universidad y la relación universidad-empresa. El análisis de las competencias tiene en cuenta, en primer lugar, la distinción entre competencias genéricas y específicas; a continuación se recogen las diferentes opiniones según el tamaño de la empresa; seguidamente se analiza los desajustes observados entre oferta y demanda; finalmente, se introduce la percepción sobre la universidad y sobre las relaciones universidad-empresa.

\section{Las competencias genéricas}

Uno de los aspectos controvertidos es el de la valoración otorgada a las competencias genéricas frente a las específicas. De las opiniones expresadas se deduce la existencia de un consenso en torno a las nuevas orientaciones de la demanda de trabajo, que consiste en poner de relieve la importancia de las competencias genéricas, antaño prácticamente ignoradas. Así pues, sin olvidar la importancia de los conocimientos técnicos o específicos básicos de la profesión, los empresarios hacen hincapié en la importancia de otro tipo de competencias (las genéricas), a menudo enraizadas en la propia personalidad, lo que está en la línea de investigaciones como Teichler (2007), Andrew y Hgison (2008), García-Aracil y Van der Velden (2008) o Tomlinson (2012). En general, destaca la alusión al compromiso, la capacidad de adaptación, de organización, de decisión, de análisis, las habilidades comunicativas y sobre todo la capacidad de trabajo en equipo. Véanse algunas opiniones al respecto:

"Las competencias que actualmente está valorando el tejido empresarial son: capacidad analítica y de adaptación, trabajo en equipo, mucha capacidad transformacional, responsabilidad y autonomía, mucha inteligencia emocional para interpretar contextos diversos..." (GD2)

"Hoy en día no se trabaja individualmente, se trabaja en equipo... deben ser responsables, saber organizarse..." (SGO)

"Cuando se incorpora una persona nueva no se debe esperar que sepa de todo... pero hay aspectos como el espíritu crítico... saber organizarse, la motivación y la inquietud para aprender, el trabajo en equipo, la capacidad de análisis y de síntesis que son esenciales, yo diría que son de manual..." (GE4)

interpersonales (capacidades individuales que facilitan la crítica e interacción social) y sistémicas (relacionadas con la visión global, de relación e integración de diversas dimensiones). 
"Las habilidades comunicativas, capacidad de interrelación, responsabilidad e implicación, capacidad de análisis y búsqueda de soluciones y visión transversal cuenta mucho..." (GE5).

Además de los atributos enunciados, también hay opiniones que destacan la importancia de otras competencias como el liderazgo, la creatividad y la visión estratégica, si bien dichas opiniones parecen estar más vinculadas a la formación de grupos de trabajo más propios de la gran empresa.

"En los procesos de selección el conocimiento se presupone... se trata de valorar otras competencias y la actitud... habilidades comunicativas, de negociación, el liderazgo..." (GE8)

"Si quisiera contratar a alguien como gestor de mi empresa le pediría que tuviera cierto liderazgo, en este papel confluyen la figura de controler, administrador y jefe de personal, con capacidad para aportar ideas, de organizarse y planificar, con autonomía y capacidad de comunicación..."(GE6)

"Lo que le interesa a la empresa es si esta gente es emprendedora, creativa, autónoma, tienen capacidad de trabajo o gestión de equipos, inteligencia emocional....al final son los elementos que determinan su contratación..."(GRH)

"Los universitarios no deben poseer solo conocimientos técnicos sino además deben demostrar destrezas tales como trabajo en equipo, iniciativa, liderazgo, creatividad, capacidad analítica, capacidad de comunicación...". (GD3).

"Un titulado de ADE, aunque no tenga formación específica del sector, aporta visión económica y estratégica..."(GE7).

Se destaca, en particular, la necesidad del dominio de algunas competencias instrumentales, como los idiomas y las TIC. En cuanto a la primera, la opinión sobre la importancia del inglés es generalizada, incluso en los casos en que no sea estrictamente preciso para la actividad de la empresa; en ese sentido, se valoran positivamente los programas de intercambio internacional. A menudo, el conocimiento de idiomas se utiliza como criterio discriminador en la contratación de titulados.

"En un entorno globalizado quien no sepa inglés esta fuera del mercado y es mejor que conozca alguna lengua más...el $75 \%$ de las demandas de trabajo de titulados de ADE exigen un buen nivel de inglés..."(GRH).

"Hoy en día tener cualquier carrera y no tener un idioma es excluirse del $80 \%$ de las ofertas que salen al mercado... Hay que dominar idiomas si no quieres cerrarte puertas..." (GD2).

"En algunas áreas como el Marketing, los idiomas son fundamentales... por ejemplo yo tengo trabajando en este área un titulado de ADE que habla seis idiomas..."(GE6).

El conocimiento de las TIC, generalmente se da por supuesto; en cualquier caso, no constituye un grave impedimento para la empleabilidad, al considerarse fácilmente subsanable. 
"El dominio de los TIC es fundamental... el empresario lo da por supuesto, es una commodity... facilita la elaboración de numerosos cálculos, análisis y corrección de desviaciones..."(GE1).

"En el tema de los TIC, por ejemplo, la evolución del lenguaje es rápida, por tanto, no se trata de tener conocimientos concretos sino de que los titulados salgan con una buena base, después ya aprendrán..." (GRH).

\section{Las competencias especificas}

En este caso se incide especialmente en la demanda de los conocimientos propios de la gestión, principalmente contabilidad, finanzas y tributación. La demanda no es tan generalizada para el resto de competencias específicas, como derecho, comercio exterior o marketing, por tratarse de competencias más diferenciadas, solo exigibles en los casos en los que la empresa tiene una orientación concreta en este ámbito de actividades.

"Las universidades deben desarrollar los conocimientos básicos relacionados con la contabilidad y las finanzas y deben olvidarse de la excesiva especificidad... las empresas saben que los graduados tienen conocimientos de los conceptos... la especificidad ya la desarrollará durante su carrera profesional... Learning by doing..." (CCB).

"Para un titulado de ADE son imprescindibles conocimientos de contabilidad, control financiero, selección de inversiones... porque la aplicabilidad práctica de estos contenidos es grande..."(GD2).

"Las materias contables y su interpretación son indispensables... Nosotros no solo necesitamos que sepan dónde se carga o abona una partida, sino también por qué se hace... la gente debe dominar también los temas financieros..."(GE4).

"Un licenciado debe tener una visión global, claro, pero debe conocer y dominar áreas específicas... para mí la contabilidad y las finanzas son las más importantes si estamos hablando de gestión... también habría que tener nociones de derecho laboral, fiscal y mercantil... porque ya recurrirán a técnicos externos si los necesitan..."(GE6)

\section{Diferencias de requerimientos por tamaño de las empresas}

En cuanto a la tipología de la empresa, merece la pena resaltar las diferencias observadas entre las demandas competenciales de la mediana y gran empresa, por una parte, y las demandas de microempresas y pequeñas empresas, por otra. Desde el punto de vista de las primeras se pone más énfasis en aquellas actitudes o competencias que permiten integrar más fácilmente al titulado en el equipo de trabajo de la empresa. En ese sentido, se prioriza la transversalidad y la carrera profesional del graduado. Naturalmente, esto se explica por el hecho de que la empresa dispone de los mecanismos suficientes para conseguir que el nuevo contratado pueda asumir todo el 
bagaje de conocimiento, tácito y explícito, que la empresa ha acumulado y que constituye lo que suele conocerse como su "cultura empresarial". Así, el graduado es un aspirante a engrosar el capital humano colectivo de la empresa, si bien la empresa no depende a corto plazo de la capacidad de trabajo del nuevo contratado.

"A la empresa le interesa más que el graduado tenga ciertas competencias, porque los aspectos técnicos, si faltan, pueden incorporarse más tarde. Cuando la empresa es grande tiene su propio dispositivo de formación porque trabaja con métodos e instrumentos específicos. Además, en un mundo profundamente cambiante los conocimientos técnicos también lo son..." (SGO).

"Conocer un programa informático... saber el número de un asiento de memoria...ya lo aprenderás... yo lo que quiero es que analicen los expedientes, que sean críticos, capaces de interpretar, de aprender y hacer consideraciones... no se les puede pedir que hagan un informe perfecto el primer día... esto es igual, ya lo aprenderán a medida que integren la cultura organizativa de la empresa..." (GE4).

"En una gran empresa... hay una experiencia colectiva (un capital social). Por lo tanto, si entra alguien nuevo habrá que enseñárselo..."(GE8).

"El crecimiento de la empresa requiere una mayor visión estratégica e iniciativa..." (GE10).

El punto de vista de las microempresas y de las pequeñas empresas, por el contrario, tiende a estar más condicionado por las características de la actividad que realizan y por la inmediatez de sus necesidades a corto plazo. En efecto, el esfuerzo relativo realizado por parte de estas empresas en la contratación de un titulado es mucho mayor que en el caso de las empresas de mayor tamaño, lo que significa que su dependencia del capital humano contratado es mayor. Por lo tanto, este tipo de empresas pone más énfasis en competencias de orden más práctico, como la multidisciplinariedad y las competencias específicas que conllevan conocimientos de aplicación inmediata y práctica para la empresa.

"Estas empresas pequeñas de 20 o 30 trabajadores no pueden pagar especialistas en todos los campos. Lo que necesitan es multidisciplinariedad, es decir, que el graduado sepa hacer, que pueda trabajar en más de una campo, en dos o en tres: contabilidad, marketing, finanzas..." (GD2)

"En el caso de pymes... deben tener habilidades transversales que les permitan pasar de una actividad a otra de la empresa... en las pymes hoy hablas de caja, mañana de ingresos, de recursos humanos... tocas muchas teclas... ...la cadena jerárquica es más corta y te piden veinticinco mil cosas a la vez..." (GE1)

"Una pyme no es un bonsái de un gran empresa, no se trata de tenerlo todo a pequeña escala; es diferente, requiere individuos versátiles, que puedan desarrollar la profesión ya y, sobre todo, multidisciplinariedad..." (PIMEC)

"La tipología de microempresas y pequeñas empresas es diversa pero, generalmente, el graduado contratado debe realizar operaciones variadas...y hacerlo inmediatamente..." (GE3). 
"Las pequeñas empresas no tiene capacidad de espera... valoran que sepan hacer lo que deben saber hacer, lo que la empresa necesita para sacar el trabajo hoy y no dentro de seis meses..." (GE2).

"Para la pequeña empresa es más relevante la polivalencia y la resolución de problemas... que el graduado tenga un buen background"(GE9)

En ocasiones, las necesidades inmediatas de las PYME se plasman en opiniones que tienden a exigir incluso formación ad hoc.

"Si más del $80 \%$ de los titulados acaban utilizando, por ejemplo, un programa de contabilidad, la universidad tiene obligación de proporcionarles las herramientas de aprendizaje..." (PIMEC)

"Los graduados normalmente suelen tener problemas de adaptación, les cuesta organizarse, planificar, trabajar en grupo y realizar un informe o cualquier otra valoración.... Yo lo que digo es que si el método de trabajo necesita estas aptitudes, su conocimiento y dominio debería ser una competencia a adquirir..." (GE2).

\section{Los desajustes observados entre oferta y demanda}

La relación entre formación teórica y formación práctica es uno de los principales motivos de desajuste entre oferta y demanda de trabajo de los graduados. La percepción que tienen los participantes en el estudio sobre la formación universitaria es de un exceso de contenidos teóricos, en ocasiones poco actualizados, y una escasa orientación práctica. Se trata de un déficit difícil de solventar, ya que enfrenta la formación, habitualmente generalista, de la universidad, con la práctica, normalmente específica, de cada empresa; en consecuencia, el período de adaptación o aprendizaje es inevitable. En este sentido, de acuerdo con Andrews y Hgison (2008), DuPre y Williams (2011) y Montoro et al. (2012) los empleadores valoran positivamente la experiencia profesional y los programas de prácticas en empresas.

"Las universidades deberían tomar conciencia de que han de incorporar en sus estudios elementos más prácticos, de adecuación a la realidad y a las necesidades del tejido empresarial actual..."(GD3)

"Sorprende ver personas con curriculum muy buenos... que no han trabajado la gestión de un asiento contable... Deberían ver soportes contables y aplicarlos. Conceptualmente lo conocen pero no saben aplicarlo..."(GE2)

"Los graduados a menudo no saben cómo combinar las cosas, no saben que, en muchos casos, están conectadas entre sí, no tienen visión sistémica.. que es muy necesaria para proyectarse en una compañía..." (GE1)

"La universidad da poco contenido práctico... esto es algo que siempre he dicho, la gente sale de allí con unos conocimientos teóricos... pero que no saben aplicar cuando aterrizan en la empresa..."(GE2)

"Cualquier tipo de experiencia de trabajo es apreciada....es importante reforzar el curriculum con prácticas empresariales..." (GRH) 
"A la universidad le falta práctica... hoy en día las prácticas empresariales son muy valoradas..." (GE5)

En relación con lo anterior, también se observan déficits en competencias genéricas, algunas estrechamente relacionadas con el aprendizaje mediante la práctica, lo que denota, según los entrevistados, el mencionado desajuste formativo.

"Las capacidades personales están muy valoradas, percibiéndose un nivel inferior a lo que se necesitaría... sobretodo la capacidad de interrelación, la multidisciplinariedad, capacidad analítica, las habilidades comunicativas y el trabajo en equipo... El tema de las conocimientos técnicos se da por supuesto..." (GE5)

"Nosotros esperamos que tengan iniciativa, determinación, que saquen reflexiones, que se atrevan a cruzar índices que puedan interesar a la empresa y hagan propuestas... pero les falta capacidad de organización y capacidad de análisis e interpretación de la información empresarial..."(GE1)

"Ese manejo de datos y reflexión, de analizar y de sacar conclusiones les cuesta, posiblemente es un tema de edad, de posición de contratante, pero la carencia es real y probablemente ligada a como reciben la formación en la universidad..."(GE6)

Desde un punto de vista general, también se aprecia cierto grado de sobrecualificación. Ello indica la existencia de un posible desajuste entre el rendimiento educativo esperado de la titulación (a través de la retribución salarial) y la productivitat laboral realmente desempeñada en el puesto de trabajo. De acuerdo con la información recogida, la sobrecualifación es un fenómeno cada vez más habitual. En efecto, para algunos autores (Freeman, 1976; García-Montalvo, 2005; Clark et al., 2014) el origen de la sobrecualificación reside en el exceso de oferta de graduados, lo cual permitiría al sector empresarial una supuesta maximización de la relación competencias versus costes de contratación. En otras palabras, dado que el salario depende en mayor medida de las características del puesto de trabajo que de las características del trabajador, en situaciones de exceso de oferta de trabajo cualificado, la competición por la vacante laboral se salda utilizando el nivel de estudios como atributo relevante de la contratación, lo que, por otra parte, puede comportar una deficiente asignación de recursos.

“...Se ve mucha gente con licenciaturas, grados y masters... sin trabajo o en ocupaciones de categorías poco relevantes dada su formación... esto implica que hay muchas cosas en el sistema que no funcionan... son temas no solo vinculados a la evolución de la economía sino también al sistema educativo..." (SGO)

"Al final resulta que en función de la demanda existente, la oferta acaba adaptándose y no al revés... nosotros pensamos que la universidad tiene un sesgo... nuestra forma de proveer la educación universitaria lleva a que se contraten personas con una formación superior a la requerida... se confunde nivel académico con capacidad para desempeñar determinados trabajos... por ejemplo si necesito un 
administrativo no debería contratar a un titulado en ADE... no están calificados para desarrollar tareas de este perfil, tienen carencias técnicas..." (PIMEC).

"Demandar perfiles más altos de los necesarios es más habitual de lo que debería ser... hay menos demanda pero más exigente... ahora los empresarios lo queremos todo, que sean jóvenes, dinámicos, que sepan hacer y aprender, que tengan idiomas y disponibilidad de viajar... y todo por el módico precio de 1000 euros..."(GE5)

"A veces te demandan cubrir puestos de trabajo que no precisan de un titulado en ADE... pero, tanto por tanto zapatos grandes.... esto me parece desaprovechar talento... "(GE2) .

"A menudo los problemas que acucian a la microempresa y pequeña empresa les lleva a intentar que la contratación de un graduado se convierta en la panacea que resolverá dichos problemas, lo que puede derivar en frustración..." (GE3)

\section{Percepción de la universidad y de la relación universidad-empresa}

Además de la valoración de las competencias, también realizaron reflexiones sobre la formación universitaria. En general, la percepción que los agentes entrevistados tienen de la universidad es de cierta lejanía, ya que la relación universidad-empresa tiene lugar más en el ámbito institucional que en la actividad cotidiana. Esto se refleja en el desconocimiento de los planes de estudio, objetivos y orientación de la universidad, de los que tienen una noción indirecta y parcial, incorporada fundamentalmente a través de los titulados que acceden al mercado de trabajo. En consecuencia, a partir de esta experiencia y de la preocupación por la adecuación entre la oferta de trabajo y los requerimientos de la demanda, los empresarios consideran que sus aportaciones deberían ser relevantes en los procesos de discusión sobre la formación de los graduados y el diseño de las titulaciones, destacando la conveniencia de aproximar la universidad a la empresa y de incorporar su presencia en aquellos niveles en los que puede establecerse un intercambio de información y opiniones sobre las actividades y necesidades de cada institución:

"Paradójicamente, para la universidad el tema competencial es secundario, prima la transmisión de conocimientos técnicos... y los procesos de reclutamiento son justamente a la inversa..." (SGO)

"Creo que hay un primer punto/déficit... las universidades no tienen la información suficiente sobre cuáles son las necesidades del mercado de trabajo o de los diferentes sectores... ...aunque se ha avanzado un poco: todas las universidades tienen sus consejos profesionales....continua faltando un canal de comunicación estable ..." (SGO)

"La realidad es muy compleja, tenemos el cajón de las titulaciones a las que se asignan unos contenidos y competencias... Y por otra el cajón del mundo del trabajo que puede necesitar otro tipo de formación... debería preocuparnos si la formación encaja o no con las ofertas de trabajo disponibles..." (PIMEC) 
"Nosotros lo que pedimos es poder canalizar una vía activa de participación del sector productivo con la universidad, participar en lo que debería hacerse....nos gustaría poder transmitir lo que desearíamos de un titulado..." (GE1)

"Se necesitan cambios y reflexión generalizados... la oferta de plazas universitarias, por ejemplo, se determina en función de la capacidad instalada y no en función de las necesidades de la sociedad..." (GD1)

En esta línea argumental, se realizan propuestas de cambio de la enseñanza universitaria que se resumen, básicamente, en dos alternativas. Ambas tienden a incidir en la orientación profesionalizadora de la actividad universitaria, aunque con distinto énfasis y enfoque. Por una parte se considera que la universidad debería ser la antesala de la actividad profesional, con todos los aspectos que ello conlleva en cuanto a realismo de la actividad y su inclusión en las aulas. Por otra parte, se demanda el retorno a una universidad más generalista, criticando la excesiva proliferación de titulaciones y defendiando el consiguiente retorno a una sólida formación en los fundamentos de cada disciplina.

"Hoy en la universidad hay un debate abierto sobre cuáles son sus funciones. De hecho hay una parte de la universidad que se horroriza de que ésta debe tener un perfil más profesionalizador........si bien es cierto que la universidad debe ir más lejos que eso,esta claro que una parte de los estudios debería ser más profesionalizadora ..." (PIMEC)

"Creo que la universidad ... debería ser mucho más práctica, más profesionaliadora, enfocada a la formación de graduados adaptados a la realidad que vivimos... ¿de qué sirve tener el grado de ADE o Economía si no sabe cómo aplicar los conocimientos adquiridos...., tomar decisiones, orientar el camino que la empresa debería seguir para sobrevivir?..." (GE)

"La excesiva especialización es un error... ahora hay mucha gente que sabe mucho de determinados campos pero no tiene visión transversal... yo diría que esta visión transversal del profesional es fundamental..."(GE5)

"Creo que la gente sale preparada para hacer cosas, pero no para resolver problemas...no es buena la excesiva especialización. Siempre he creído que las carreras han de ser generalistas, pero eso va en contra de la corriente actual." (G8).

Se incide, también, en la necesidad de cambiar la metodología de enseñanzaaprendizaje

"El rodillo de la Universidad debería preparar a los estudiantes para que supieran reaccionar en distintos escenarios o situaciones por dos motivos, uno para que fueran mucho más versátiles y dos para que tuvieran posibilidad de proyección. Esto debería implicar decisiones de cambios en el material y en el método de enseñanza y de estudio..." (GE1)

"Es necesario comenzar a trabajar en equipos interdisciplinarios y establecer colaboraciones entre la universidad y el sector productivo de manera que se 
faciliten los cambios de modelo y se introduzcan metodologías más activas que faciliten la adquisición de competencias transversales..." (GD1)

\section{Discusión y conclusiones}

El análisis realizado muestra que el concepto de competencias profesionales juega un papel central en la demanda de características de los graduados por parte de los empleadores, es decir en la empleabilidad. Además, aun reconociendo la importancia de las competencias específicas, se enfatiza en la demanda de competencias genéricas. Así, los empleadores utilizan su desarrollo competencial para discriminar entre los candidatos a un mismo puesto de trabajo.

Los resultados ponen de manifiesto que la valoración de las competencias genéricas y específicas no es igual para todo tipo de empresas. En efecto, para las microempresas y pequeñas empresas es más importante la inmediatez de resolver los problemas cotidianos que la visión a largo plazo y, por ende, que la consolidación de la carrera profesional del graduado, lo que les lleva a valorar la capacidad de aplicación inmediata de los conocimientos, a exigir que la persona contratada "sepa hacer" más que "tenga capacidad de aprender"; en definitiva valoran más las competencias específicas que las genéricas. Por el contrario, las medianas y grandes empresas tienden a conceder más valor a las competencias genéricas, priorizan "la flexibilidad y la capacidad de aprendizaje"; se plantean una estrategia a largo plazo y adaptan la formación a dicha visión. En el fondo de esta divergencia se encuentra una diferencia tecnológica en la organización y funcionamiento de ambos tipos de empresas: mientras que las grandes empresas tienen un bagaje organizativo y de funcionamiento amplio, es decir, una amplia "cultura empresarial", capaz de absorber nuevos elementos que la enriquezcan; las microempresas y pequeñas empresas a menudo carecen de todo el bagaje tecnológico lo que imposibilita dicho planteamiento estratégico.

En la línea de autores como Alonso et al. (2008), Marzo et al. (2009), Montoro et al. (2012) y Martín del Peso et al. (2013), también se evidencian desajustes en las competencias desde el punto de vista de la oferta formativa de los graduados y la demanda de requerimientos en cuanto a conocimientos, actitudes y habilidades de los empresarios. Los principales déficits se observan en la formación práctica y en el desarrollo de algunas competencias genéricas, como la motivación, las habilidades relaciones, la capacidad iniciativa, de adaptación, de análisis o la capacidad de organización. Asimismo, parece observarse cierto grado de sobrecualificación, que parece haberse agudizado durante el período de recesión. Este desajuste, que supone una ineficiente asignación de capital humano, sin embargo, no es visto como un inconveniente desde el punto de vista de los empleadores, en la medida en que puede asimilarse a la existencia de una oferta de elevada calidad y, por tanto, de una supuesta maximización de la contratación.

En definitiva, la existencia de desajustes entre oferta y demanda nos remite al concepto de asimetrías informativas e introduce incertidumbre en el proceso de contratación. En este contexto, las titulaciones pierden relevancia como indicador de valoración obligando a los empleadores a incorporar nuevos instrumentos de 
referencia. En consecuencia, se hace necesario introducir cambios en el modelo de enseñanza-aprendizaje, así como una mayor aproximación de las relaciones universidad-empresa. Si bien no hay una clara solución institucional a este problema, se observan acciones encaminadas en ese sentido. Entre dichas acciones cabe destacar el esfuerzo de las universidades en el fomento de las prácticas de estudiantes mediante convenios de colaboración con empresas, la creación de oficinas de carreras profesionales, ferias de ocupación, recreación de escenarios de aprendizaje práctico, el fomento de actividades de internacionalización, etc. Este tipo de actuaciones favorece el encuentro entre ambos ámbitos y contribuye a reducir las asimetrías de información entre empleadores y graduados, mejorar la capacidad competencial de los graduados y facilitar con ello los ajustes oferta-demanda en el mercado de trabajo.

La integración de la universidad en el tejido productivo, que se desprende de la opinión de los empleadores, no está exenta de controversia. Para algunos autores (Bock, 2003; Bolivar, 2008; Corominas y Sacristán, 2011) esta perspectiva utilitarista subordina la universidad al mercado. Ello contradice la finalidad que tradicionalmente le ha sido asignada, más acorde con una formación integral de los individuos, en la que la formación para el mercado de trabajo debería constituir una dimensión más del proyecto formativo. No obstante, ello no significa que la universidad no deba permanecer en continua reflexión sobre los cambios, tanto en el ámbito del conocimiento, como en el de su transmisión y aplicación.

\section{Referencias bibliográficas}

ABDULLAH, A, KAMAL, N. Y NUNA, S.(2014). Employability Factors of Business Graduates in Kuwait: Evidence from An Emerging Country, International Journal of Business and Management, 9 (10), 49-61.

ALONSO, L, FERNÁNDEZ, C.J. Y NYSSEN, J.M. (2008). El debate sobre las competencias. Una investigación cualitativa en torno a la Educación Superior y el Mercado de trabajo en España. Madrid: ANECA.

ANDREWS, J. Y HIGSON, H. (2008). Graduate employability, "soft skills" versus "hard" business Knnowledge: A European study. Higher Education in Europe, 33 (4), 411-422.

BIEMANS, H., NIEUWENBUIS, L., POELL, R., MULDER, M. Y WESSELINK, R. (2004). Competence based VET in the Neherland. Background and piftalls. Journal of Vocational Education and Training, 56 (2), 523-538.

BECKER, G. (1964). Human Capital. New York: Columbia University Press.

BOCK, D. (2003). Universities in the marketplace: the commercialization of Higher Education. Princenton: University Press.

BOLIVAR, A. (2008). El discurso de las competencias en España: educación básica y educación superior. Revista de docencia universitaria, monográfico 2, 1-23. 
BROWN, A. (2010). Qualitative method and compromiso in applied social research. Qualitative Research, 10 (2), 229-248.

CLARK, B., JOUBERT, C. Y MAUREL, A. (2014). The career prospects of overeducated Americans. NBER Working Paper $\mathrm{N}^{\mathrm{O}} 20167$.

CORBETTA, P. (2010). Metodología y técnicas de investigación social. Madrid: McGraw-Hill.

COROMINAS, A. Y SACRISTÁN, V. (2011): La encrucijada estratégica de la universidad pública española. Revista de Educación, 355, 57-81.

COROMINES, E., SAURINA, D., VILLAR, E. (2010). Adequació de la formació universitària al mercat de treball. Anàlisi de tres cohorts de graduats a Catalunya. Barcelona: AQU.

FREEMAN, R. (1976). The Overeducation Amerincan. New York: Academic Press.

DUPRE, C. Y WILLIAMS, K. (2011). Undergraduates' perceptions of employer expectations. Journal of Career and Technical Ecucation, 26(1), 8-19.

FREIRE, M.; TEIJEIRO, M. Y PAIS, C. (2013). La adecuación entre las competencias adquiridas por los graduados y las requeridas por los empresarios. Revista de Educación, 362, 13-41.

GARCÍA-ARACIL, A. Y VAN DER VELDEN, R. (2008). Competencies for young European higher education graduates: Labour market mismatches and their payoffs. Higher Education, 55 (2), 219-239.

GARCÍA-MONTALVO, J. (2005). La inserció laboral dels universitaris: la qüestió de la sobrequalificació i el desajust formatiu. Nota d'Economia, 81 (1), 99-119.

GONZÁLEZ, J. Y WAGENAAR, R. (ed) (2003). Tuning Educactional Structures in Europe. Deusto y Groningen: Universidad de Deusto y Universidad de Groningen

HECKMAN, J., STIXRUD, J. Y URZUA, S. (2006). The Effects of Cognitive and Noncognitive Abilities on Labor Market Outcomes and Social Behavior. Journal of Labor Economics, 24 (3), 411-482.

MARTÍN-DEL-PESO, M., RABADÁN-GÓMEZ, AB., HERNÁNDEZ-MARCH, J. (2013). Desajustes entre formación y empleo en el ámbito de las Enseñanzas Técnicas Universitarias: La visión de los empleadores de la Comunidad de Madrid. Revista de Educación, 360, 244-267.

MARZO-NAVARRO, M., PEDRAJA-IGLESIAS, M., RIVERA-TORRES, P. (2009). Curricular profile of University graduates versus business demands: Is there a fit or mismatch in Spain?. Education and training, 15 (1), 56-69.

MINCER, J. (1958). Investment in Human Capital and Personal Income Distribution. Journal of Political Economy, 46 (4), 281-302.

MONTORO SÁNCHEZ, M.A., MORA VALENTÍN, E.M. Y ORTIZ DE URBINA, M. (2012). Análsis de las competencias adquiridas en los estudios de Dirección de 
Empresas y su grado de aplicación en las practicas en empresas. Revista Complutense de Educación, 23 (1), 242-263.

PATTON, M. (2015). Qualitative evaluation and research methods. Newbury Park: Sage.

PÉREZ, F. (2005). La entrevista como técnica de investigación social. Fundamentos teóricos, técnicos y metodológicos. Extramuros, 8. 187-210.

POLANY, M. (1958). Personal Knowledge: Towards a Post-Critical Philosophy. Chicago: University of Chicago Press.

RODRÍGUEZ, R. (2015). Competencias genéricas en la enseñanza superior a través de los programas de Internacionalización. Revista Complutense de Educación, 26 (1), 81-100.

ROMER, P. (1990). Endogenous Technical Change. Journal of Political Economy, 98( 5),. 71- 102.

SCHOMBURG, H., Y TEICHLER, R,U. (2006). Higher Education and Graduate Employment in Europe. Results of Graduates Surveys from 12 Countries. Dordrecht: Springer.

SCHULTZ, T. (1961). Investment in Human Capital. American Economic Review, 51, $1-17$.

SPENCE, M. (1973). Job Market Signaling. The Quarterly Journal of Economics, 87 (3), 355-374.

TEICHLER U. (2007). Does Higher Education Matter?. Lesson from a Comparative Graduate Survey. Journal of Education, 42 (1), 11-34.

TEICHLER, U. Y VAN DER VELDEN, R. (eds.) (2000). Higher Education and Graduate Employment. European Journal of Education, 35 (2) (special issue).

THUROW, L. (1983). Un modelo de competencia por los puestos de trabajo, en M. J. Piore, (comp), Paro e inflación : perspectivas institucionales y estructurales, 57-76. Madrid: Alianza Editorial.

TOMLINSON, M. (2012). Graduate Employability: A Review of Conceptual and Empirical Themes. Higher Education Policy, 25, 407-431.

TRULLÉN, J. LLADÓS, J. BOIX, R. (2002). Economía del conocimiento, ciudad y competitividad. Investigaciones Regionales, 1, 139-161.

VELA, F. (2001). La entrevista cualitativa: una puerta de entrada a la realidad social. En M.L. Tarrés (coord.), Observar, Escuchar y comprender sobre la tradición cualitativa en la investigación social, 63-95. México: Facultad Latinoamericana de Ciencias Sociales.

\section{Anexo: Glosario}


Abreviaturas introducidas en el texto.

CCB: representante de la Cámara de Comercio de Barcelona

GE: empresario participante en el grupo de empresarios entrevistados

- $\quad \mathrm{GE} 1=$ pyme del sector servicios (restauración);

- GE2=pyme del sector servicios (consultoría fiscal, contable, laboral, recursos humanos),

- $\quad$ GE3=pyme del sector servicios (consultoría- análisis empresas);

- GE4= representante de la Secretaría de Ocupación de la Generalitat de Cataluña.

- GE5= ex directivo de gran empresa; actualmente director de una pyme de ingeniería;

- GE6=pyme industrial (producción y comercialización de productos cosméticos);

- $\quad \mathrm{GE7}=$ gran empresa del sector servicios (hostelería y restauración);

- GE8=gran empresa del sector farmacéutico (producción y comercialización).

- GE9=pequeña empresa del sector tecnológico.

- $\mathrm{G} 10=$ gran empresa tecnológica.

GD: participante en el grupo de discusión

- $\mathrm{GD} 1=$ representantes del ámbito académico

- $\quad \mathrm{GD} 2=$ representantes del ámbito empresarial

- $\quad$ GD3=representantes de la Administración Pública.

GRH: gestor de recursos humanos (servicios de colocación internacional)

PIMEC: representante de la asociación de pequeñas y medianas empresas de Cataluña

SGO: representante de la Secretaria General de Ocupación de la Generalitat de Cataluña

\section{Correspondencia con los autores}

$\mathrm{M}^{\mathrm{a}}$ Teresa BARTUAL FIGUERAS

Departament de Teoria Econòmica

Universitat de Barcelona.

Av Diagonal, 696. $4^{\text {a }}$ Planta.

08034 Barcelona.

e-mail: bartual@ub.edu

Joaquín TURMO GARUZ

Departament de Teoria Econòmica, Universitat de Barcelona.

Av Diagonal, 696. $4^{\mathrm{a}}$ Planta.

08034 Barcelona.

e-mail: jturmo@ub.edu 